and with how to express uncertainty. Although patients were mostly able to recall what the doctor had said, they often interpreted particular terms (such as mild) differently from what was intended by the clinician. Successful and unsuccessful reassurance seemed to hinge on the patient's perception that the doctor had understood and acknowledged his or her current difficulties and indicated this using appropriate and acceptable terminology.

In conclusion, this study suggests that patients may be successfully reassured if clinicians avoid loaded terms such as "mild" and "early stages" and try to acknowledge patients' perspectives that their difficulties are serious. Attempting to reassure patients in this way might seem to require more time, but we found that it was the perception of having symptoms and problems acknowledged that seemed to matter, not more time itself.

We thank Bill Fleming for support, the clinicians who participated in this study, and the patients who contributed so much information. The Department of Social Medicine at the University of Bristol is the main centre for the MRC Health Services Research Collaboration.

Contributors: DB initiated the study, and JD developed the design of the study. JD carried out the interviews, observations, and data analysis and acts as guarantor. JD and DB wrote the paper.

Funding: Arthritis and Rheumatism Council.

Competing interests: None declared.

1 Fitzpatrick R. Telling patients there is nothing wrong. BMJ 1996;313: $311-12$.
2 McDonald IG, Daly J, Jelinek VM, Panetta F, Gutman JM. Opening Pandora's box: the unpredictability of reassurance by a normal test result. BMJ 1996;313:329-32.

3 Pilowsky I. Abnormal illness behaviour. Br J Med Psychol 1969;42:347-51. 4 Mayou R. The nature of bodily symptoms. Br J Psychiatry 1976;129:55-60.

5 Fitzpatrick R, Hopkins A. Referrals to neurologists for headaches not due to structural disease. J Neurol Neurosurg Psychiatry 1981:44:1061-7.

6 Bartlett EE, Grayson M, Barker R, Levine DM, Golden A, Libber S. The effects of physician communication skills on patient satisfaction, recall and adherence. J Chron Dis. 1984;37:755-64.

7 Francis V, Korsch B, Morris M. Gaps in doctor-patient communication N Engl J Med 1969;280:535-8.

8 Patient compliance. Ann Pharmacotherapy 1993;27:S5-24.

9 Henbest RJ, Stewart M. Patient-centredness in the consultation: does it really make a difference? Fam Pract 1990;7:28-33.

10 Kessel N. Reassurance. Lancet 1979;i:1128-33.

11 Freidson E. Profession of medicine. New York: Dodd Mead and Co, 1970.

12 Mishler E. The discourse of medicine. Norwood, New Jersey: Abler, 1984

13 Silverman D. Communication and medical practice. London: Sage, 1987.

14 Blaxter M. The causes of disease: women talking. Soc Sci Med, 1983;17: $59-69$

15 Williams SJ, Calnan M. Modern medicine: lay perspectives and experiences. London: UCL Press, 1996.

16 Eyles JD, Donovan JL. The social effects of health policy: experiences of health and health care in contemporary Britain. Aldershot: Avebury, 1990.

17 Donovan JL, Blake DR, Fleming WG. The patient is not a blank sheet: lay beliefs and their relevance to patient education. $\mathrm{Br} J$ Rheumatol 1989;28:58-61.

18 Donovan JL. Patient education and the consultation-the importance of lay beliefs. Ann Rheum Dis 1991;50:418-21.

19 Donovan JL, Blake DR. Patient non-compliance: deviance or reasoned decision-making? Soc Sci Med 1992;34:507-13.

20 Hammersley M, Atkinson P. Ethnography. London: Tavistock, 1983.

21 Glaser BG, Strauss AL. The discovery of grounded theory. Chicago: Aldine, 1967.

22 Mays N, Pope C. Qualitative research in health care. London: BMJ Publishing, 1996.

23 Miles MB, Huberman EM. Qualitative data analysis. 2nd ed. London: Sage, 1994.

24 Donovan JL. Patient decision making: the missing ingredient in compliance research. Int J Technol Assess Health Care 1995;11:443-55.

(Accepted 13 December 1999)

\title{
Open access follow up for inflammatory bowel disease: pragmatic randomised trial and cost effectiveness study
}

\author{
J G Williams, W Y Cheung, I T Russell, D R Cohen, M Longo, B Lervy
}

\section{School of \\ Postgraduate \\ Studies in Medical \\ and Health Care, \\ Morriston Hospital, \\ Swansea SA6 6NL \\ J G Williams \\ director \\ W Y Cheung \\ lecturer \\ B Lervy \\ senior lecturer in \\ general practice \\ Department of \\ Health Sciences \\ and Clinical \\ Evaluation, \\ University of York, \\ Heslington, York \\ YO10 5DD \\ I T Russell \\ professor of health \\ sciences \\ continued over}

BMJ 2000;320:544-8

\begin{abstract}
Objective To evaluate whether follow up of patients with inflammatory bowel disease is better through open access than by routine booked appointments. Design Pragmatic randomised controlled trial. Setting Two district general hospitals in Swansea and Neath, Wales.

Participants 180 adults (78 with Crohn's disease, 77 ulcerative or indeterminate colitis, 25 ulcerative or idiopathic proctitis) recruited from outpatient clinics during October 1995 to November 1996.

Intervention Open access follow up according to patient need.

Main outcome measures Generic (SF-36) and disease specific (UK inflammatory bowel disease questionnaire UKIBDQ) quality of life, number of primary and secondary care contacts, total resource use, and views of patients and general practitioners. Results There were no differences in generic or disease specific quality of life. Open access patients had fewer day visits $(0.21 v 0.42, \mathrm{P}<0.05)$ and fewer outpatient visits ( $4.12 v 4.64, \mathrm{P}<0.01$ ), but some patients had difficulty obtaining an urgent appointment. There were no significant differences in
\end{abstract}

specific investigations undertaken, inpatient days, general practitioner surgery or home visits, drugs prescribed, or total patient borne costs. Mean total cost in secondary care was lower for open access patients $(\mathrm{P}<0.05)$, but when primary care and patient borne costs were added there were no significant differences in total costs to the NHS or to society. General practitioners and patients preferred open access.

Conclusions Open access follow up delivers the same quality of care as routine outpatient care and is preferred by patients and general practitioners. It uses fewer resources in secondary care but total resource use is similar. Better methods of ensuring urgent access to outpatient clinics are needed.

\section{Introduction}

Gastroenterology is a busy medical specialty with a large and expanding outpatient workload. ${ }^{1}$ Many patients with gastrointestinal disorders have chronic relapsing disease and some, particularly those with inflammatory bowel disease, are traditionally kept under continuing follow up. This reflects the wishes of general practitioners ${ }^{2}$ as well as specialists, who feel 
that the unpredictable course, complications, and treatment of inflammatory bowel disease merit specialist care. ${ }^{3}$ However, this traditional approach puts increasing pressure on outpatient clinics.

The aim of the study was to evaluate open access rather than routine booked appointments as a means of following up patients with inflammatory bowel disease. Our null hypothesis was that outpatient follow up of patients with inflammatory bowel disease through open access is no worse than by routine booked appointments, as judged by health related quality of life, total resource use, and patient and general practitioner preference.

\section{Participants and methods}

The study was undertaken at two neighbouring hospitals which differ in organisation and management. Morriston is a large district general hospital which provides most regional specialties. Neath is a smaller hospital with a busy medical intake but no acute surgical services. The hospitals are nine miles $(14.5 \mathrm{~km})$ apart and between them serve a local population of about 250000 in a predominantly urban area. Gastroenterology clinics at Neath are dedicated to the specialty, whereas at Morriston the clinics also cover general medicine. Neath has a comprehensive clinical information system supporting clinical and service management which facilitates monitoring and review of patient progress. ${ }^{45}$ This was not available at Morriston.

The study was approved by the West Glamorgan local research ethics committee, and all patients gave written consent after an oral and written explanation.

\section{Protocol}

Comprehensive guidelines for the shared management of inflammatory bowel disease were distributed to all local general practitioners before the study started. These covered diagnosis, medical treatment of mild to severe disease, laboratory monitoring, the place of surgery, stoma care, follow up and surveillance, communication, documentation, and audit. For patients due for follow up by open access we transferred responsibility for care back to the general practitioner and stopped routine appointments at outpatient clinics. In return, we guaranteed rapid access to specialist care when necessary. The normal recall system continued for patients needing regular surveillance by colonoscopy because of the increased risk of colorectal cancer.

Patients were recruited by three consultant gastroenterologists, one staff doctor, two senior registrars, and four registrars from outpatient clinics at the two hospitals during October 1995 to November 1996. Patients aged over 18 with inactive or mildly active but stable inflammatory bowel disease were invited to take part. Those with active disease requiring treatment, a stoma, other disease which required regular follow up, or who were thought unable to comply with data collection were excluded. Three general practitioners declined to collaborate, and their patients were also excluded.

Patients randomised to routine follow up made their next appointment at the end of each hospital visit as usual. Our practice in both hospitals has been to see patients at short notice between appointments if requested by the patient or general practitioner. It was made clear to patients in the routine arm that this policy still applied. Those randomised to follow up through open access were asked to contact their general practitioner about problems or to contact the hospital directly if they were unable or unwilling to see the general practitioner first. Appointments were made by telephoning outpatient clerks or gastroenterology secretaries, who were made aware of the need to offer an early appointment.

Patients were reviewed in the outpatient clinic in the normal way. A relapse did not require withdrawal, but the patient was seen and treated as appropriate and remained in the same study group. All patients were called for review 24 months after entry into the study.

Primary outcome was measured by the generic and disease specific quality of life questionnaires SF- $36^{67}$ and UK inflammatory bowel disease questionnaire (UKIBDQ). ${ }^{8}$ The questionnaires were completed in clinic at recruitment and at the end of the study and by post at six monthly intervals between. Two reminders were sent to non-respondents. Those who failed to attend the end of study appointment after two reminders were sent the final questionnaire by post.

Resource use was estimated from patient questionnaires and case notes. Medical staff abstracted data from hospital notes, and practice staff abstracted them from general practitioner records. Questions on patient borne costs within the quality of life questionnaires covered travel, parking, time off work for the patient and any accompanying person, and other costs such as baby sitting. Patient and general practitioner satisfaction, preferences, and views were assessed by postal questionnaires at the end of the study, supplemented by semistructured interviews with a sample of general practitioners. To minimise bias these were undertaken by general practitioners during audit visits.

When the trial was designed no disease specific health related quality of life scale had been validated for use in the United Kingdom. As we found no previous study of inflammatory bowel disease which used SF-36 as an outcome, we estimated sample size using SF-36 scores from patients with suspected peptic ulcer, ${ }^{7}$ which showed a standard deviation of 20 for most subscales. We considered a difference of 10 points to be clinically important, equivalent to a standardised difference of 0.5 . Hence, 170 patients (85 per group) would yield $90 \%$ power to detect a significant difference with a significance level of $0.05 .{ }^{9} \mathrm{~A}$ target of 180 was set to allow for loss to follow up. Limited information on the distribution of key resource variables meant that the sample size could not reflect likely differences in costs. ${ }^{10}$

Analysis was by intention to follow up. To counteract the effect of possible differences in baseline health related quality of life scores, we analysed changes in individual scores from baseline using $t$ tests. Preference data were analysed by $\chi^{2}$ tests.

Because data on use of resources tend to be highly skewed, routine parametric statistics are not appropriate. We therefore assessed significance by the Mann-Whitney U test. As economic analysis is mainly concerned with a comparison of means, however,
Business School, University of Glamorgan, Pontypridd CF37 1DL D R Cohen professor of health economics M Longo research officer

Correspondence to: J G Williams john.williams@ pgms.wales.nhs.uk 


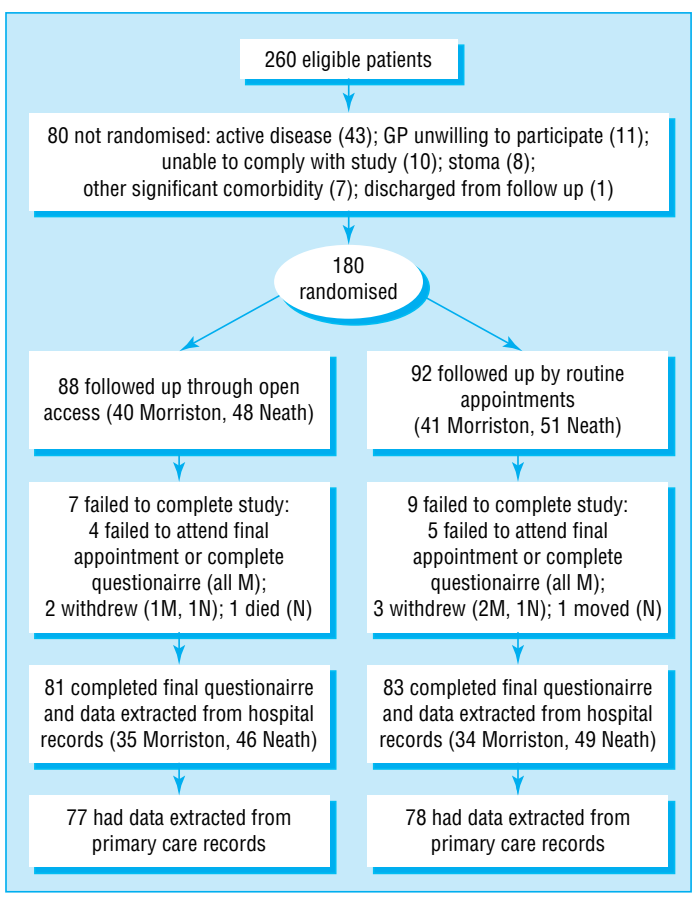

Progress of participants through trial

means and standard deviation are reported for each variable.

Valuation of hospital resources was based on estimates provided by the trusts. Costs of outpatient and general practitioner home visits were derived from Netten et al, ${ }^{11}$ drug costs from the British National Formulary ${ }^{12}$ and costs of general practitioner surgery visits from Graham and McGregor. ${ }^{13}$ Patients' lost work time was valued by using average wages, ${ }^{14}$ and their motoring costs were estimated from Automobile Association figures. ${ }^{15}$ Total costs to society were derived by summing primary care, secondary care, and patient borne costs.

\section{Random allocation}

To ensure balance in type of follow up, patients were first stratified by centre and between four diagnostic

Table 1 Differences between patients allocated to open access and routine follow up in changes in quality of life from baseline

\begin{tabular}{lcccc} 
& \multicolumn{4}{c}{ Mean difference $\mathbf{( 9 5 \%} \mathbf{~ C l})^{*}$} \\
\cline { 2 - 5 } & $\begin{array}{c}\text { At } \mathbf{6} \\
\text { months }\end{array}$ & $\begin{array}{c}\text { At } \mathbf{1 2} \\
\text { months }\end{array}$ & $\begin{array}{c}\text { At 18 } \\
\text { months }\end{array}$ & At 24 months \\
\hline UK inflammatory bowel disease questionnaire: & & & & \\
\hline Bowel movements and use of facilities & -0.3 & 4.5 & -0.5 & $0.3(7.7$ to -7.1$)$ \\
\hline General bowel symptoms & 0.2 & -1.3 & -1.6 & $-3.5(4.0$ to -10.9$)$ \\
\hline Emotional function & -1.1 & -0.0 & -1.6 & $-1.3(3.4$ to -5.9$)$ \\
\hline Social function & -1.3 & 0.5 & -2.4 & $0.4(6.5$ to -5.7$)$ \\
\hline Systemic function & -2.8 & 2.6 & -0.3 & $2.2(9.9$ to -5.6$)$ \\
\hline SF-36: & & & & \\
\hline Vitality & 0.1 & -2.5 & -6.1 & $-3.7(3.3$ to -10.7$)$ \\
\hline General health perception & -0.0 & -4.6 & -2.2 & $-3.5(2.0$ to -8.9$)$ \\
\hline Mental health & -2.2 & -0.4 & -4.1 & $-3.7(2.4$ to -9.9$)$ \\
\hline Bodily pain & 0.8 & 0.8 & -0.5 & $-2.5(5.0$ to -10.0$)$ \\
\hline Physical functioning & -0.5 & 2.0 & -6.0 & $-3.7(3.2$ to -10.5$)$ \\
\hline Role limitations due to physical problems & -8.3 & -2.7 & -2.6 & $-2.7(11.4$ to -16.8$)$ \\
\hline Social functioning & -2.9 & -2.1 & -4.2 & $-0.4(8.1$ to -8.9$)$ \\
\hline Role limitations due to emotional problems & -13.3 & -1.2 & -3.4 & $-5.3(11.4$ to -22.0$)$ \\
\hline
\end{tabular}

*Positive differences denote a better change in open access patients than in routine outpatients; negative differences denote a better change in routine outpatients than in open access patients. groups: ulcerative or idiopathic proctitis; ulcerative or indeterminate colitis affecting more than the rectum; Crohn's disease of the small or large bowel; and Crohn's disease of the small and large bowel. The computer generated allocation lists were securely held by one independent researcher in each centre. When the clinician had established the eligibility of the patient and received informed consent, the local researcher was contacted for the random assignment and the patient immediately informed of the follow up arrangements.

\section{Results}

\section{Participant flow and follow up}

The figure shows the progress of the trial. No patients refused to participate, although five subsequently withdrew. Quality of life questionnaires were completed by 170 patients at six months (94\%), 160 at 12 months (89\%), 159 at 18 months (88\%), and 164 at two years $(91 \%)$. The number of patients who failed to complete the study differed significantly between the two hospitals (12 in Morriston $v 4$ in Neath; $\mathrm{P}<0.05$ ). There was no significant difference between groups at baseline in age, sex, diagnostic group, or quality of life.

\section{Quality of life}

There was no significant change in mean health related quality of life scores in either group over the two years of the study, although there was some deterioration in both groups in most subscales. We found no significant differences between groups in changes in health related quality of life scores at $6,12,18$, or 24 months compared with baseline (table 1).

\section{Patients' preferences}

Patients had a strong preference for open access follow up $(103 / 164, \mathrm{P}<0.01) ; 69 / 81(85 \%)$ in the open access follow up group preferred open access follow up, and $34 / 83(41 \%)$ in the routine group would have preferred open access follow up. The main reason given for this preference was the appropriateness of attending only when ill. The reason most commonly given for keeping routine appointments was for reassurance. Some patients had difficulty arranging open access appointments, and a few would probably have been lost to follow up if they had not been called for the end of study visit.

\section{General practitioners' preferences}

Study patients were registered with 53 practices. Forty practices returned postal questionnaires relating to 155 patients, including 12 patients who did not complete the final patient questionnaire $(86 \%$ response rate). Sixty nine general practitioners indicated their preferred method of follow up for 143 patients (including eight who did not complete the final patient questionnaire). The general practitioners preferred open access follow up for 108 patients (55 in open access follow up, 53 routine) and routine follow up for 35 patients (15 open access, 20 routine). This difference was highly significant even after potential correlation between multiple responses from individual practitioners was allowed for $(\mathrm{P}<0.001)$. Preference for open access follow up was associated with 
sensible patients, stable disease, and the effective booking of urgent review. Forty four general practitioners (64\%) favoured a gastrointestinal nurse practitioner as point of contact; 10 were opposed to this, eight wanted further discussion of the role, and seven did not express a view.

\section{Use of resources}

Comprehensive data on resource use in both primary and secondary care was available for 155 patients. Table 2 shows use of hospital facilities. Open access patients had fewer day visits $(\mathrm{P}=0.019)$, fewer outpatient visits $(\mathrm{P}=0.002)$, and cost less in total investigations $(\mathrm{P}=0.032)$. There were no significant differences in numbers of inpatient days or specific investigations. Patient borne costs were lower for open access patients $(\mathrm{P}=0.002)$. Mean total cost for hospital care was significantly lower for open access patients than routine outpatients ( $£ 582 v £ 611, \mathrm{P}=0.012$ ).

Analysis of resource use in primary care showed no significant differences in general practitioner visits, patient borne costs, or drugs prescribed (table 3). Although more maintenance drugs (5-aminosalicylates) were prescribed in the open access follow up group, this did not reach significance. Primary care costs were higher for open access patients, but not significantly so. When primary and secondary care costs were considered together there was no difference in total costs to society between study groups (table 4).

\section{Discussion}

For patients with quiescent or mild, stable inflammatory bowel disease, open access follow up is preferred by patients and general practitioners and allows less resource intensive follow up in outpatient clinics without deterioration in quality of life. Quality of life questionnaires were completed in clinic at the beginning and end of the study, and by post at six monthly intervals in between. Completion in clinic tends to underestimate the effect of disease on quality of life,${ }^{16}$ but this would not affect our comparisons between groups.

Because resource use was skewed in both groups, a larger sample size would have been needed to detect all true differences in costs. However, we found trends toward lower secondary care costs and higher primary care costs for open access patients. In secondary care these differences were significant, even though the cost of open access follow up showed greater variability.

\section{Problems with open access}

Despite the strong preference for open access follow up, some patients experienced difficulties in making urgent appointments, largely because of pressure on clinics and the inexperience of clerical staff in managing open access follow up. Letters from general practitioners were effective but took time. We believe that the best way to overcome this problem is to have a single telephone point of contact for patients that is staffed by a specialist gastrointestinal nurse practitioner. General practitioners were generally supportive of this proposal, and we intend to introduce and evaluate this approach. Chronic inflammatory disease is a well recognised risk factor for the development of gastrointestinal malignancy. ${ }^{17} 18$ As well as managing open access, a nurse practitioner could ensure that
Table 2 Mean (SD) resources used per patient in hospitals over 24 months

\begin{tabular}{lccc} 
Resource variables & $\begin{array}{c}\text { Open access } \\
(\mathbf{n}=\mathbf{7 7})\end{array}$ & $\begin{array}{c}\text { Routine visit } \\
(\mathbf{n}=\mathbf{7 8})\end{array}$ & $\begin{array}{c}\text { P value for } \\
\text { difference }\end{array}$ \\
\hline No of outpatient visits & $4.12(3.41)$ & $4.64(2.38)$ & 0.002 \\
\hline No of day cases & $0.21(0.47)$ & $0.42(0.66)$ & 0.019 \\
\hline No of inpatient days & $0.83(3.53)$ & $0.41(1.74)$ & 0.71 \\
\hline No of investigations: & & \\
\hline Full blood count & $2.44(2.43)$ & $2.79(2.25)$ & 0.09 \\
\hline $\begin{array}{l}\text { Erythrocyte sedimentation rate and C } \\
\text { reactive protein }\end{array}$ & $2.27(2.29)$ & $2.47(2.23)$ & 0.48 \\
\hline Biochemical profile & $2.12(3.21)$ & $2.46(2.41)$ & 0.10 \\
\hline Colonoscopy & $0.17(0.38)$ & $0.31(0.54)$ & 0.09 \\
\hline Rigid sigmoidoscopy & $0.08(0.39)$ & $0.12(0.46)$ & 0.53 \\
\hline Flexible sigmoidoscopy & $0.31(0.57)$ & $0.32(0.55)$ & 0.81 \\
\hline Biopsy & $0.22(0.62)$ & $0.24(0.54)$ & 0.45 \\
\hline Vitamin B-12, folate, and ferritin & $0.49(0.91)$ & $0.57(0.99)$ & 0.58 \\
\hline Total cost of investigations $(£)$ & $198(278.99)$ & $257(276.10)$ & 0.032 \\
\hline Total cost of secondary care $(£)$ & $582(807.94)$ & $611(475.47)$ & 0.012 \\
\hline Patient borne cost $(£)$ & $74(61.72)$ & $87(47.67)$ & 0.002 \\
\hline Total cost to society $(£)$ & $656(859.74)$ & $699(516.17)$ & 0.011 \\
\hline
\end{tabular}

*Mann-Whitney U test.

Table 3 Mean (SD) resources used per patient in primary care over 24 months

\begin{tabular}{|c|c|c|c|}
\hline Resource variables & $\begin{array}{l}\text { Open access } \\
\quad(n=77)\end{array}$ & $\begin{array}{l}\text { Routine visit } \\
\quad(n=78)\end{array}$ & $\begin{array}{l}\text { P value for } \\
\text { difference }\end{array}$ \\
\hline No of surgery visits & $9.23(7.76)$ & $7.73(5.77)$ & 0.47 \\
\hline No of home visits & $0.36(1.15)$ & $0.41(1.23)$ & 0.69 \\
\hline \multicolumn{4}{|l|}{ No of tablets or enemas: } \\
\hline Immunosuppressive drugs† & $248(521)$ & $167(633)$ & 0.70 \\
\hline Maintenance drugs $\ddagger$ & $1287(1475)$ & 855 (1234) & 0.14 \\
\hline Antidiarrhoeal drugs§ & $298(838)$ & $283(1138)$ & 0.95 \\
\hline Steroid enemasף & $9(31.6)$ & $7(33.2)$ & 0.42 \\
\hline Total cost of drugs $(£)$ & $376(464)$ & $263(404)$ & 0.17 \\
\hline Total primary care costs $(£)$ & $464(467)$ & $340(431)$ & 0.07 \\
\hline Patient borne costs $(£)$ & $40(33)$ & $35(27)$ & 0.53 \\
\hline Total costs to society $(£)$ & $504(472)$ & $375(438)$ & 0.06 \\
\hline
\end{tabular}

*Mann-Whitney U test.

†Prednisolone, azathioprine.

¥Mesalazine, olsalazine, balsalazide.

§Diphenoxylate, codeine phosphate, loperamide.

१Hydrocortisone, prednisolone.

Table 4 Summary of mean (SD) costs for open access and routine follow up ( $£$ )

\begin{tabular}{|c|c|c|c|}
\hline & $\begin{array}{c}\text { Open access } \\
(\mathrm{n}=77)\end{array}$ & Routine ( $\mathrm{n}=78$ ) & $\begin{array}{l}\text { P value for } \\
\text { difference* }^{*}\end{array}$ \\
\hline Secondary care & $582(808)$ & $611(475)$ & 0.01 \\
\hline Primary care & 464 (467) & $340(431)$ & 0.07 \\
\hline Total NHS cost & $1046(948)$ & $951(680)$ & 0.89 \\
\hline Patient borne cost & $115(82)$ & $122(64)$ & 0.07 \\
\hline Cost to society & 1160 (1007) & $1074(724)$ & 0.78 \\
\hline
\end{tabular}

${ }^{*}$ Mann-Whitney U test.

patients are called back at appropriate intervals for assessment and colonoscopy if necessary.

There were no significant differences in patient characteristics between the two hospital sites. However, significantly more patients at Neath completed the study. The dedicated clinics and computerised clinical information system at Neath may have contributed to this.

As far as we are aware, this is the first randomised trial comparing open access and routine follow up for patients with inflammatory bowel disease, although Probert et al recommended such reorganisation in 1993. ${ }^{3}$ Their survey reported that most gastroenterologists in Britain cared for at least 100 patients with inflammatory bowel disease, and nearly a quarter of them for 200 or more. Thus the reduction in 


\section{What is already known on this topic}

Routine follow up of patients with inflammatory bowel disease is putting increasing pressure on outpatient clinics

Transferring the responsibility for care of patients with asthma saves resources in secondary care without increasing primary care workload or affecting patients health related quality of life

\section{What this study adds}

Open access follow up for patients with inflammatory bowel disease does not affect patient care but saves secondary care resources

Most patients prefer follow up through open access

General practitioners think open access follow up is more appropriate for most patients

Effective methods are needed for making urgent appointments

outpatient attendances which we have documented could save each consultant 25-50 visits a year.

\section{Wider applicability}

A study of shared care of patients with moderately severe asthma also found that it was equally effective as hospital care and produced cost savings in secondary care without a significant increase in primary care workload. ${ }^{19}$ Similarly, a randomised trial of patients with breast cancer showed that follow up of patients in remission by general practitioners was not associated with increased time to diagnosis of relapse, increased anxiety, or deterioration in health related quality of life. However, resource use and preferences were not evaluated. ${ }^{20}$

Although much has been written about shared care, ${ }^{19-24}$ further studies are needed to evaluate whether our findings can be extrapolated to conditions such as arthritis, epilepsy, heart failure, and multiple sclerosis. Much would depend on the ability and willingness of general practitioners to shoulder the increased responsibility.

Contributors:JGW conceived the study, developed the protocol, obtained funding, recruited patients, validated data collected, oversaw the conduct of the study, drafted and edited the paper, and acts as guarantor for the paper; WYC helped develop the protocol, drafted and refined questionnaires, validated data collected, undertook the qualitative and quantitative analysis, and helped write and edit the paper; ITR helped develop the protocol, provided methodological and statistical advice, and edited the paper; DRC helped develop the protocol, provided methodological advice, advised on analysis of health economics data, and helped write and edit the paper; ML analysed the health economics data and edited the paper; BL contributed to the development of the protocol, helped liaise with primary care, interviewed colleagues and edited the paper. Dr Paul Duane, Dr Jerry Kingham, Mr Chandra Sekaran, Dr David Parker, Dr Mark Whitehead, Dr Tom Yapp, Dr Mohamed Hanif, Dr Siân Morgan, and Dr Charlie Richardson helped recruit patients, supported administratively by the staff of the Clinical Research Unit at Morriston, and Mrs Teryl Stoneman at Neath, who also distributed and collated the health related quality of life questionnaires. Gary Barton helped develop the protocol and pilot health economics data collection. Dr John Dove, Dr Barbara Weatherill, and Dr Bridget Kirsop interviewed colleagues in primary care. Gaynor Demery provided clerical support. Dr Mesbahur Rahman, Dr Krishnaraj Raganuth, and Julia Demery extracted data from secondary care records, and Jayne Morgan, Hayley Dickinson, and Susie Lucas supported electronic data capture and retrieval on GeneCIS.

Funding: NHS Research and Development Primary/ Secondary Care Interface Programme with supplementary support from the West Wales and Swansea Group of the National Association for Colitis and Crohn's Disease. The Iechyd Morgannwg Health Authority Medical Audit Advisory Group allowed interviews with general practitioners to be undertaken as part of practice audit visits.

Competing interests: None declared.

1 Sellu D. Have we reached crisis management in outpatient clinics? BMJ 1998:316:635-6

2 Moody GA, Mann R, Gay S, Wicks ACB, Mayberry JF. The gastroenterology service: a survey of general practitioners' requirements. JR Soc Med 1993;86:26-7.

3 Probert CS, Jayanthi V, Mayberry JF. British gastroenterologists' care profile for patients with inflammatory bowel disease: the need for a patients' charter. J R Soc Med 1993;86:271-2.

4 Williams JG, Morgan JM, Severs MP, Howlett P. Let there be light. $\mathrm{Br} J$ Health Care Computing 1993;10:30-2.

5 Williams JG. The use of clinical information to help develop new services in a district general hospital. Int J Med Inf 1999;56:151-9.

6 Jenkinson C, Coulter A, Wright L. Short form 36 (SF36) health survey questionnaire: normative data for adults of working age. BMJ 1993;306:1437-40

7 Garratt AM, Ruta DA, Abdalla MI, Buckingham KJ, Russell IT. The SF36 health survey questionnaire: an outcome measure suitable for routine use within the NHS? BMJ 1993;306:1440-4

8 Cheung WY, Garratt A, Russell IT, Williams JG. The UKIBDQ: a British version of the inflammatory bowel disease questionnaire. J Clin Epidemiol (in press).

9 Altman DG. Statistics and ethics in medical research III. How large a sample? BMJ 1979;282:1336-8.

10 Drummond MF. Economic analysis alongside controlled trials. London: Department of Health, 1994.

11 Netten A, Dennett J, Knight J. Unit costs of health and social care. Canterbury: Personal Social Services Research Unit, University of Kent, 1998.

12 British Medical Association, Royal Pharmaceutical Society of Great Britain. British national formulary. London: BMA, RPS, 1998. (No 35.)

13 Graham B, McGregor K. What does a GP consultation cost? Br J Gen Pract $1997 ; 47: 170-2$.

14 Office for National Statistics. The new earnings survey. London: Stationery Office, 1998.

15 Automobile Association. Motoring costs. London: AA, 1998.

16 Lyons RA, Wareham K, Lucas M, Price D, Williams JG, Hutchings HA SF-36 scores vary by method of administration: implications for study design.J Public Health Med 1999;21:41-5.

17 Stewenius J, Adnerhill I, Anderson H, Ekelund GR, Floren CH, Fork FT, et al. Incidence of colorectal cancer and all cause mortality in non-selected patients with ulcerative colitis and indeterminate colitis in Malmo, Sweden. Int J Colorectal Dis 1995;10:117-22.

18 Bernstein D, Rogers A. Malignancy in Crohn's disease. Am J Gastroenterol 1996;91:434-40

19 Grampian Asthma Study of Integrated Care (GRASSIC). Integrated care for asthma: a clinical, social, and economic evaluation. BMJ 1994;308:559-64.

20 Grunfeld E, Mant D, Yudkin P, Adewuyi-Dalton R, Cole D, Stewart J, et al Routine follow-up of breast cancer in primary care: randomised trial. BMJ 1996;313:665-9.

21 Diabetes Integrated Care Evaluation Team. Integrated care for diabetes: clinical, psychosocial and economic evaluation. BMJ 1994;308:1208-12.

22 Petrie JC, Robb OJ, Webster J, Scott AK, Jeffers TA, Park MD. Computerassisted shared care in hypertension. BMJ 1985;290:1960-2.

23 Van Damme RAE, Drummond NA, Beattie JAG, Douglas JG. Integrated care for patients with asthma: views of general practitioners. Br J Gen Pract 1994;44:9-13.

24 Hickman M, Drummond N, Grimshaw J. The operation of shared care for chronic disease. Health Bull 1994;52:118-26.

(Accepted 1 February 2000)

\section{Endpiece}

\section{Machiavelli on clinical management}

He who neglects what can be done for what ought to be done, sooner effects his ruin than his preservation.

Machiavelli

Submitted by David Stone, consultant cardiologist, Papworth Everard, Cambridgeshire 